\title{
APORTES DE UNA CIENCIA MATEMÁTICA A LA INVESTIGACIÓN EN PSICOLOGÍA: algunas reflexiones desde la docencia*
}

\section{CONTRIBUTIONS OF A MATHEMATICAL SCIENCES TO PSYCHOLOGY RESEARCH: Reflections from the classroom}

\author{
Orual Andina \\ Instituto de Estadística Aplicada, Facultades de Ingeniería y Tecnologías y de Ciencias Empresariales, \\ Universidad Católica del Uruguay

\section{Daniel Sucazes} \\ Instituto de Estadística Aplicada, Facultades de Psicología y de Ciencias Empresariales, \\ Universidad Católica del Uruguay
}

\footnotetext{
* Ponencia presentada por el Prof. Daniel Sucazes en ocasión del 3er Simposio de Investigación en Psicología de la Facultad de Psicología de la UCU, Montevideo, 2010.
}

\section{INTRODUCCIÓN}

En general, se reconoce como una experiencia común a todos los profesores de Estadística Aplicada a la Psicología, la de encontrarse al inicio de cada curso, con un grupo de estudiantes capaces y entusiastas, muy interesados en la psicología, que no solamente no tienen ni idea de lo que es la Estadística ni para qué sirve, sino que además necesitan sobreponerse a un serio bloqueo emocional antes de comenzar a comprenderla. La pregunta que a todos se les ocurre, pero que muy pocos se atreven a formular es, ¿qué tiene que hacer una ciencia matemática, entre los cursos obligatorios exigidos para alcanzar el grado de Licenciado/a en Psicología?.

Antes que nada, es necesario aceptar que la duda es completamente legítima. Durante siglos -probablemente hasta el siglo XVII- existía solo una ciencia matemática de lo cierto y de lo inmutable. Los modelos construidos hasta entonces solo permitían explicar los llamados experimentos determinísticos, en los que se tiene razonable certeza con respecto a la ocurrencia de un resultado individual. Era la matemática que necesitaban, por ejemplo, la física y la química para desarrollarse y progresar como ciencias.
Prácticamente en el otro extremo, se encontraban -entre otras- las llamadas ciencias humanas. Estas ciencias rehuian todo contacto con la matemática determinística, que -con razón- consideraban inaplicable al material con el que tenían que trabajar, caracterizado por la variabilidad y la incertidumbre. Era mucho más razonable buscar un mayor apoyo en la literatura o en la filosofía.

Recién a partir del siglo XVII lo incierto y aleatorio comenzó a dejar de ser una fuerza oscura y misteriosa, a partir de los hallazgos logrados por matemáticos de la talla de Pascal, Bernoulli, Laplace, Gauss y Poisson, entre otros. Ellos demostraron que también se podían construir modelos matemáticos para lograr explicaciones razonables de lo no determinístico. Los primeros biómetras y estadísticos, como Galton y Pearson, fueron los que comenzaron con la aplicación de estos nuevos modelos matemáticos a las ciencias humanas. Hacia 1900, el mismo Pearson y también Spearman, realizaron aportes decisivos a la ciencia estadística aplicada a la investigación en Psicología. Desde entonces y con muy raras excepciones, la Estadística comenzó a formar parte indispensable de los programas de todas las carreras de Psicología que imparten las universidades de mayor prestigio. 
Probablemente, la duda todavía subsiste entre nuestros estudiantes, porque en la enseñanza pre-universitaria del Uruguay no se ha insistido mucho acerca de la existencia de esta, ya no tan nueva, matemática (lamentablemente tampoco sobre la otra). De manera que es frecuente encontrar en nuestro país, estudiantes universitarios (no sólo de Psicología) que al no tener un gusto especial por los números, creen que es posible prescindir -casi por completo- de ellos.

Actualmente, la secuencia clásica del conocimiento científico (formulación de la hipótesis - experimento - prueba de la hipótesis) se ha integrado a la investigación en Psicología con la misma importancia que en las ciencias físicas y biológicas o en economía, sociología, educación, medicina y agricultura. Por lo tanto se requiere tener un conocimiento cabal de dónde se quiere llegar, si el material experimental es suficientemente representativo; saber sobre la mejor forma de diseñar el experimento y ejecutarlo y asegurar que, en todo el proceso, se cumplan las condiciones necesarias para poder aplicar la teoría del muestreo y el cálculo de probabilidades. Es así que, para poder investigar en Psicología es necesario tener un buen conocimiento de la teoría estadística y suficiente dominio de sus aplicaciones prácticas a la realidad de interés específico.

Los investigadores en Psicología deben saber que la estadística, como todas las ciencias matemáticas, tiene total validez lógico formal, es decir que está constituida por elementos abstractos enteramente demostrables en base a ciertos axiomas originados en el descubrimiento y confirmación de cierta permanencia, estabilidad o regularidad en la presentación de un conjunto de resultados experimentales. Sobre estos axiomas se construyen -por pura deducción lógica- una serie de proposiciones, lemas, teoremas, etc., que en su conjunto constituyen lo que usualmente se denomina, una ciencia matemática.

Para la construcción de la Teoría de la Probabilidad, que es el sustento matemático de la Estadística, se sigue este mismo proceso. Los resultados de los llamados "experimen- tos aleatorios" son tan irregulares, que no es posible predecir con certeza un resultado individual. Sin embargo, si un experimento aleatorio se repite en condiciones esencialmente iguales, es posible observar cierta regularidad en los resultados, identificar axiomas y construir modelos matemáticos que permitan explicar el comportamiento de este tipo de experimentos; utilizamos entonces el cálculo de probabilidades.

Afortunadamente, las reglas de una ciencia matemática como la Estadística por su sola mención, se aceptan como razonables. La comprobación empírica de la validez de estas reglas, en innumerables ejemplos del mundo real, contribuye a fortalecer y a perfeccionar una fuerte base intuitiva, que permite admitir una regla matemática como verdadera sin ayuda de la razón. Sin embargo, se requiere tener un buen conocimiento de la estructura lógico formal de la estadística. En las aplicaciones de la estadística a la investigación, no existen reglas fijas. En la mayoría de los casos, la elección de un modelo teórico-conceptual adecuado a una situación empírica no es una tarea sencilla, especialmente cuando se requiere su modificación o adaptación para lograr una mejor adecuación a las condiciones que se presentan. Hay que tener en cuenta que, la validez conceptual de una ciencia matemática queda comprobada si es posible demostrar los enunciados con base en los axiomas de la teoría. Sin embargo, la estadística es también una ciencia aplicada. Es decir que resulta necesario demostrar que, su aplicación práctica al mundo real, produce resultados satisfactorios.

Actualmente, existe abundante software especializado para el análisis estadístico. Pero hay que tener en cuenta que las grandes facilidades operativas que ofrecen, no pueden reemplazar en modo alguno las etapas de un conocimiento tan profundo como sea posible de los fundamentos teórico-conceptuales de la ciencia, así como de la metodología que los relaciona con las diferentes aplicaciones prácticas. Consecuentemente, hay que tener cuidado con una combinación que suele producir resultados desastrosos: alta tecnología en manos inexpertas. 


\section{LA SITUACION ACTUAL}

Debemos reconocer que desde un principio, la ciencia Estadística tuvo una alta prioridad en los programas de estudio de la carrera de Psicología de la Universidad Católica del Uruguay. En todos estos años, ese interés por la Estadística Aplicada a la Psicología se ha ido reforzado en forma muy significativa, como lo demuestra el énfasis que las autoridades de la Facultad han puesto en la investigación psicológica, en la preferencia que se otorga a la investigación para realizar los trabajos de grado, en la creación de la Revista "Ciencias Psicológicas" y la fundación del Laboratorio "Alberto Cha" (en merecido reconocimiento a la labor del distinguido docente).

En la actualidad, se reconoce la importancia de brindar oportunidades de reforzamiento a la capacitación y perfeccionamiento en estadística, a los interesados en la investigación en el área, que con bastante frecuencia encuentran limitaciones importantes a la hora de recoger datos, procesarlos, analizarlos y difundirlos, por la insuficiencia de su capacitación en métodos y procedimientos estadísticos. En los cursos de pregrado, las asignaciones horarias dedicadas a la estadística, son generalmente insuficientes, por lo tanto, los programas de la asignatura se restringen a aspectos introductorios, sin que los estudiantes puedan lograr un suficiente dominio de las aplicaciones prácticas específicas, basados en sus propias experiencias. Respondiendo a esas necesidades, las autoridades universitarias tuvieron la iniciativa de crear un Instituto de Estadística Aplicada que, entre otras finalidades, debería ocuparse de diseñar, planificar y ejecutar un programa sostenible de capacitación y perfeccionamiento en esa área. Entre otras propuestas, este instituto, que ya se encuentra en funciones, ha elaborado un proyecto de Diploma en Estadística Aplicada, actualmente en estudio.

\section{LA ENSEÑANZA DE ESTADISTICA EN LOS CURSOS DE PSICOLOGÍA}

Los estudiantes de Psicología (entre otras carreras) suelen no tener afinidad alguna por la Estadística; muchos de ellos -y en particular los que esperan dedicarse a la Psicología Cínica- se sienten afligidos por encontrarse al comienzo del plan de estudios con una asignatura que, creen innecesaria para el desempeño de la profesión. Sin embargo, a medida que avanzan en la carrera van comprendiendo la necesidad de interiorizarse en los conceptos, métodos y procedimientos estadísticos. Vencer el bloqueo inicial resulta una tarea verdaderamente difícil, especialmente porque la aversión a los números comienza a tomar forma en edades tempranas y va creciendo y haciéndose cada vez más fuerte a medida que avanzan en sus cursos de enseñanza media. Afortunadamente existen estudiantes que no presentan síntomas de sufrir este bloqueo. Pero lo asombroso de la situación es que cada año éstos representan una proporción cada vez más pequeña del total.

Uno de los posibles caminos para vencer esta dificultad, consiste en establecer ciertas condiciones que identifiquen a los cursos de Estadística como producto útil, de buena calidad, que posibilitan al estudiante enfrentar nuevos problemas, porque ha creado una forma de razonamiento aplicable a la solución de los actuales y adaptable a las nuevas exigencias que se planteen en el futuro.

Esas condiciones se resumen en, presentar una exposición conceptual clara, sencilla pero sin pérdida de rigurosidad; efectuar una aplicación inmediata a casos frecuentes en la investigación, con el objetivo de que los estudiantes comprendan la utilidad de la herramienta y el empleo de criterios de evaluación justos y objetivos, que permitan valorar adecuadamente el rendimiento, promoviendo el reconocimiento de logros.

Se debe insistir en la ejercitación práctica, para que los estudiantes logren seleccionar los elementos conceptuales más adecuados para cada situación, puedan efectuar los cálculos con fluidez y finalmente, tomar las decisiones que cada caso particular amerite. El fruto logrado con el esfuerzo propio, permite generar la confianza necesaria para continuar adelante. Afortunadamente, es bastante frecuente la experiencia de recibir comentarios de los estudiantes confesando su aversión inicial por los números, los cálculos, las gráficas...; hasta que la participación en un trabajo de investigación les permitió comprender su significado y su importancia para describir la realidad y posibilitar las mejores decisiones. 
Los docentes deberíamos poder generar en los estudiantes un sano sentido estadístico de ver la realidad; comprendiendo que el mismo es importante para cualquier persona y que, como estudiantes o como profesionales, siempre habrá ocasiones en que tendrán necesidad de consultar informes de trabajos de investigación científica y entender su terminología y conclusiones -expresadas en lenguaje matemático- y también que, si desean describir los resultados obtenidos al aplicar determinado tipo de intervención o efectuar comparaciones con otros resultados obtenidos con tratamientos alternativos, será imprescindible contar con herramientas estadísticas apropiadas.
Esta ponencia ha tenido como objetivo presentar -muy brevemente- por un lado, la historia de la inclusión de la Estadística en la formación básica del Profesional en Psicología y por otro - y como docentes- reflexionar sobre las creencias con que inicia la carrera el estudiante de grado en Psicología. Finalmente, se exponen algunas consideraciones sobre los aspectos que se entienden son relevantes en la formación de profesionales y especialmente, de investigadores en Psicología. 